\title{
Everything in a Part: About the Creation of Universe and Consciousness
}

\section{Di Sia $\mathrm{P}^{1,2 *}$ and Bhadra $\mathrm{NK}^{3}$}

${ }^{1}$ Department of Chemical Science and Neuroscience, University of Padova, Italy

${ }^{2}$ Faculty of Science and Technology, Free University of Bolzano, Italy

${ }^{3}$ Lakshmipur Swamiji Seva Sangh High School Laskhmipur, Gobardanga, India

\section{Conceptual Paper}

Volume 4 Issue 1

Received Date: February 07, 2020

Published Date: February 27, 2020

DOI: $10.23880 /$ eoij-16000228

*Corresponding author: Paolo Di Sia, Department of Chemical Science, School of Science,

University of Padova, Via Marzolo 1, 35131 Padova, Italy, Tel: 00393402668170; Email: paolo.disia@gmail.com

\section{Abstract}

In this paper we consider a scenario beyond the well known Standard Model (SM) of physics that is holistic, appropriate for the explanation of everything, including the consciousness. Mathematically we deal with the Super Unified Gaussian Energy Group SU(11) instead of SU(5) related to SM. The presence of the group SU(6) inside SU(11) can allow, after the symmetry breaking of SU(11), the creation of new particles, responsible of the consciousness. Consciousness can be considered as a non-local quantum information, describing an elegant information-participatory universe connecting quantum-information consciousness and physics; so we can understand consciousness, energy and matter as different expressions of a same informational order. Very important is the function of the entanglement, assuring an informational universe-consciousness interconnection. A recent model in progress, said B-DS model, is part of this global scenario, in the attempt to contribute in explaining reality in its totality in a holistic way, leaving the scheme that sees contemporary physics focused only on matter, with the exclusion of consciousness.

Keywords: Consciousness; Matter; Universe; Contemporary Physics; Entanglement; Vacuum Structure; Quantum Information; B-DS model

\section{Introduction}

We are to date quite familiar with how matter behaves. Its behaviour is dictated by the laws of physics, in particular quantum physics. Classical physics works also very well for objects of macroscopic dimensions; quantum mechanics is instead necessary in the microcosmos, allowing phenomena who seem completely weird at classical level, as the entanglement.

During years, the fact that classical mechanics was not able to account various phenomena, or parts of them, vehiculated the research to use quantum features. The idea that quantum mechanics can help for plants and some bacteria (let us think to recent studies about photosyntesis, quantum biology, quantum chemistry) brought to reflect to it in relation to bigger and more complex organisms [1].

In recent years enzyme catalysis, photosynthesis and avian navigation have been quantum biology's most popular applications; similarly, research is looking for the quantum underpinnings of smell, the origin of consciousness and of life itself, asking if quantum effects are regular features of these systems or if nature actively uses them to improve how things are done. It seems to be therefore that living systems are not devoid of quantum effects even outside carefully controlled lab experiments [2].

It has been considered that the human brain and its mental aspects are associated with classical brain physiology 
and are also part of a quantum physical universe. The human brain is conceived as an interfacing organ for mind and consciousness and also receiving information. The brain or its parts is conceived as an interference medium of incoming data and already existing data, which are equivalent to the subject's memory [3].

A recent model in progress, said B-DS model, is investigating in holistic way the reality, taking benetifs by phenomena such as the entanglement and paying close attention to the structure of the vacuum [4].

\section{Technical Aspect of this New Model}

We assume that our physical universe appeared by a symmetry breaking, a generalized Gaussian energy group, the Super Unified Gaussian Energy Group SU(11) of 10-dimensional space-time, bringing then to the 4-dimensional Einstein's universe of Grand Unified Theories (GUTs) described by SU(5), our present Standard Model [5].

This class of symmetry groups can be mathematically expressed by using Lie-algebra as:

$$
\begin{aligned}
& \mathrm{SU}(5) \supset \mathrm{SU}(3) \times \mathrm{SU}(2) \times \mathrm{U}(1), \quad(1) \\
& \mathrm{SU}(11) \supset \mathrm{SU}(5) \times \mathrm{SU}(6) \times \mathrm{U}(1), \quad(2) \\
& \mathrm{SU}(23) \supset \mathrm{SU}(11) \times \mathrm{SU}(12) \times \mathrm{U}(1), \quad(3) \\
& \mathrm{SU}(47) \supset \mathrm{SU}(23) \times \mathrm{SU}(24) \times \mathrm{U}(1), \quad(4)
\end{aligned}
$$

and so on. It is considered that the breakdown of Super Unified Theory (SUT) symmetry group SU(11) breaks into fundamental groups $S U(5) \times S U(6)$, leading to a phase-like transition, and then SU(5) breaks into subgroups SU(3) $\times$ $\mathrm{SU}(2) \times \mathrm{U}(1)$.

We think that in the early universe something similarlike to the Curie-temperature for ferromagnetism happened to the Super Unified Theory of SU(11). Above like a critical temperature Tc the vacuum state, the state of lowest energy, was the potential $\Phi=0$. Below Tc, the states of lowest energy of the thermo-statistical particles were changed. It now corresponds to a situation when $\Phi$ has non-zero values. The consequences of this, for the very early universe, are that it was divided into different domains, each with a different value of $\Phi$. In this way the universe acquired discontinuities along the domain walls, bringing to highly significant discontinuities of matter distribution. The fact that we do not see such discontinuities is currently known as the "domain wall problem" [6].

In gauge theories in physics, the special unitary group SU(n) is very important; in theories of symmetry breaking, it is significant to find its subgroups. Important subgroups of $\mathrm{SU}(\mathrm{n})$ for the physics of unification are $\mathrm{SU}(\mathrm{p})$ and $\mathrm{SU}(\mathrm{n}-\mathrm{p})$, with $\mathrm{p}>1, \mathrm{n}-\mathrm{p}>1$. It holds:

$$
\mathrm{SU}(\mathrm{n}) \supset \mathrm{SU}(\mathrm{p}) \times \mathrm{SU}(\mathrm{n}-\mathrm{p}) \times \mathrm{U}(1) .(5)
$$

From the symmetry breaking of SU(11), we find the subgroups $S U(6)$ and $S U(5)$, where $p=5, n-p=6$, so that Eq. (5) is satisfied.

From SU(11) we have 120 arbitrary constants, corresponding to 120 bosons; 24 are related to SU(5), 35 to $\mathrm{SU}(6)$ and 1 to U(1). Other 60 bosons are needed to make up the list of 120 . For want of any specific designation, we refer to them as the "J-bosons". The J-bosons are expected to link the participants of SU(6) with SU(5), i.e. emission and absorbtion of J and anti-J particles. Therefore, in the theory of SU(11), it is possible to change any of 30 latent energy bosons of SU(6) into any of the 30 matter energy bosons of SU(5) or vice-versa, by the exchange of the J-bosons of $\operatorname{SU}(11)[7,8]$.

\section{On the Essence of the Vacuum}

The development of quantum mechanics provided a description of reality going often beyond any classical logic and forecast. One of the most important results, still tied to date to unresolved problems, is the existence of a vacuum energy. In classical electrodynamics there is no reason why there should be electromagnetic radiation in a vacuum; but already in 1912 Max Planck, studying the black body spectrum, found a "zero point energy", starting from the hypothesis that the radiation was made up of discrete quanta of energy. From quantum theory, in particular from the quantization of the electromagnetic field, emerged again the vacuum energy.

In vacuum, the vector potential and electromagnetic fields satisfy the D'Alembert's equation and therefore they are represented in terms of superposition of polarized plane waves. Solving the wave equation for the vector potential within a cubic volume, the Hamiltonian field coincides with that of a system of infinite decoupled harmonic oscillators. Each frequency of oscillation of the electromagnetic field is represented by a harmonic oscillator, which is a "normal mode". Using the correspondence principle, the electromagnetic field can be quantized and, through the "creation" and "annihilation" operators, it is possible to represent the energy in terms of discrete quantities, the "photons" [9].

A discrete spectrum for the field energy emerges, corresponding to that of infinite harmonic oscillators, and non-zero fluctuations of the ground state, the "state of vacuum". The origin of the zero point energy lies in the noncommutability of the creation and annihilation operators and, by means of the uncertainty principle, vacuum fluctuations can be associated with virtual photons that annihilate each 
other [10]. The appearance in quantum electrodynamics of vacuum energy represented a turning point of theoretical physics, since it is responsible for the divergence of the Hamiltonian of any system with infinite degrees of freedom.

If, from a formal point of view, the vacuum energy can be eliminated through a normal rearrangement, its presence in purely quantum phenomena such as Lamb shift and Casimir effect cannot be ignored. This resolution opened the way to quantum field theory (QFT).

The Lamb shift provides a correction to the fine structure of the hydrogen atom, involving the vacuum fluctuations of the inner electromagnetic field. The Casimir effect is a phenomenon of quantum nature but manifesting itself in macroscopic reality, appearing when boundary conditions to the quantized electromagnetic field are imposed; it is a force exerting between two flat "infinite" perfectly conductive plates. This interaction is the macroscopic manifestation of the vacuum fluctuations constrained by the material boundaries of the physical system under examination $[11,12]$.

The Casimir effect manifests itself also on the atomic scale, causing the attraction between two atoms or nearby neutral molecules (Casimir-Polder force). The experimental verification of these two phenomena came several years after their theoretical formulation. The Lamb shift was determined with the help of spectroscopy; the Casimir effect has been confirmed by significant experiments, but the study around it is still subject of debates and discussions in the scientific community [13].

\section{Examples of Resonant Containers}

The evolution is first and foremost a matter of harmonic patterning in a containment field, regardless of whether the container is a body or a structured space. It is a well-known fact that the sound produces regular geometric patterns when particles of powder are vibrating on plates or inside liquid containers. Researchers such as Ernst Florens Friedrich Chladni and Hans Jenny have shown how vibrated waves reflect in containers to form circles, triangles, pentagons, hexagons and other, more elaborate, mandala-like patterns $[14,15]$.

As waves, they resonate into standing waves with different frequencies combining to form regular patterns by crossing one another at whole number proportions. The same thing can be said to occur in the atomic substratum of DNA as in cell mitosis. In this way, living cells would dynamically selforganize into stable geometries, like powder vibration inside a spherical water container.
In the container of a human body, the energy of resonating atoms, molecules and cells could be considered as a reflected standing wave, rippling outward from the 12 x $2=24$ vertebrae of the spinal column with less and less energy to the tips of our $5 \times 2$ fingers and $5 \times 2$ toes. The body results therefore described as a $12: 5$ dodecahedral proportioned carbon water crystal, resonating into the "empty space" container, a lattice created by the new energy sources within the theory of the Super Unified Gaussian Energy Group SU(11) [1]. The in-depth study of the structure of the vacuum will provide further answers to the still not resolved questions.

\section{On the Importance of Holographic Principle and Entanglement}

It has been proposed that entropy is the information and complexity content of a system, and not the disorder of it [16]. Developments of the quantum theory of gravity with quantized space-time demonstrated that the relation "entropy-area" is holographic-type, and it has been called the "holographic principle". Quantum entanglement is a form of information and space-time would be a manifestation of quantum information [17].

It has been proposed a model of universe as network of holograms, with each hologram containing the information of all the others, with any surface area in space becoming a channel of information. The area would be a measure of the capacity to carry information, with the space as channel of information among observers [18].

Juan Martin Maldacena developed a theory describing gravity in a volume of space-time with the number of degrees of freedom of the volume $V$ scaling as the area $A$ of the surface enclosing the same volume, known as "Maldacena duality" or "gauge/gravity duality" [19,20].

In relation to the work of Einstein showing that black holes could be connected by wormholes through space-time (known also as "Einstein-Rosen bridges") [21], Maldacena demonstrated that wormholes would form only if black holes are quantum-entangled. This suggests and implies that the entanglement (quantum effect) is linked to space-time (theory of relativity), confirming that the two main pillars of modern physics, quatum theory and relativity, are connected and not irreconcilable. Then space-time, deformable by the presence of matter, would be also a geometrical manifestation of entanglement. Moreover the information measured by entropy is related to both quantum mechanics and gravity, showing again the possible compatibility of the two theories.

Contemporary physics is therefore showing that the 
universe can be considered as a "quantum holographic entangled reality", with quantum information as key property behind it. The entanglement of the quantum-holographic consciousness with the fabric of space-time can then explain one of the greatest mistery of quantum physics, i.e. the wavefunction collapse by the observer consciousness [22].

\section{Quantum Information and Entanglement in the Fabric of Space-Time}

Information and consciousness become then an intrinsic non-local property of the universe, able of generate selforganization, order, complexity. Quantum information becomes more fundamental than energy, matter and space-time, stating that reality originates from information [23]. We live in an interconnected universe filled with a field of quantum entangled information, interpenetrating everything and everyone. David Bohm developed a non-local theory of the universe organized by non-local information with a quantum potential carrying active information. There is an implicate order unfolding in an explicate order, i.e. the manifested space-time universe, and then still enfolding in the implicate order through the holomovement. He proposed later also a superimplicate order as a function of the wavefunction, that organizes the order in complex and stable structures. Matter, energy and consciousness can be considered as expression of a same informational order [24].

The holographic theory of consciousness of Karl Pribram showed experimentally that the fields of electromagnetic activity in the brain cortex are quantum holographic nonlocal informational distributed patterns, demonstrating that receptive fields in cortical units are wavelet-like patterns as Gabor elementary functions (by Gabor studied for detecting the "unit of communication"), that in this case define processes in the material brain $[25,26]$; so the same mathematical formulation describes both. Information becomes unfolded into ordinary space-time appearance at the realization of a potential, and inversely. There is a bijective relation between non-local informational organization of the quantum-holographic universe and quantum-holographic organization of our brain-mind.

\section{Conclusion}

Energies of SU(6) would be responsible of gravitational forces which are required for the formation of a complete body with definite shape for animate and inanimate bodies, so as stars with its planets, galaxies and so on. The so called "vacuum space" would be properly filled with the energies of SU(6); in fact, what we consider "full" around us (and inside our bodies) is actually "empty", and vice-versa.

Galaxies within our universe seem to be not isolated, but connected by a sort of spiderweb-like structure permeating the space between galaxies, with the galaxies being nodes on that web. Using space telescopes, web's filaments are identified as being comprised of hot gases, mostly hydrogen. It would be the result of the nature of space itself, with pairs of particles and anti-particles that are constantly created and annihilated. The rapid expansion of space (inflation) would have prevent that, causing discrepancies in the density of the universe.

The recent scientific advances are changing our understanding of the universe, its organization and the emergence of complexity. Considering ideas from various disciplines (biology, physics, mathematics, computer science, genetics, neurology), new theories on the source of the creative power of the universe are developing, considering the collective and holistic properties of physical systems and the self-organization, a project ("blueprint") progressively unfolding as the universe develops, with the possibility of an underlying purpose.

Going beyond the Standard Model of physics, i.e. considering the symmetry theory of SU(11) and beyond (SU(23), SU(47), etc.), we are able to describe living and non living matter, adding the consciousness with the blueprint for the future developments. In the theory of SU(11) we can speculate about the definition of unknown new particles, with different strength, and several electromagnetic forcefrequencies in the framework of $S U(6) \times U(1), S U(12) \times U(1)$, $S U(24) \times U(1)$, etc., responsible for consciousness of the living cells after the creations of the electrodynamics group $\mathrm{U}(1)$.

The study of the vacuum structure is one of the avantgarde themes both in the field of theoretical physics, as well as in experimental physics. Vacuum fluctuations are not a simple mathematical result, but have a physical reality, have then a role in macroscopic world too. Experimental and theoretical research around the Casimir effect and vacuum energy offers wide-ranging perspectives, with applications in nanotechnology (in particular the field of nano-neuroscience [27-29]), chemistry and biophysics, up to the horizons of cosmology and theories of everything, finding everything in a part and vice-versa.

\section{References}

1. Di Sia P, Bhadra NK (2020) Origin of living matter by a new model of consciousness. World Scientific News (WSN) 143: 67-78.

2. Blankenship RE, Engel GS (2010) Long-lived quantum coherence in photosynthetic complexes at physiological temperature. Proceedings of the National Academy of Sciences of the United States of America 107(29): 
12766-12770.

3. Di Sia P, Bhadra NK (2019) Mind and Consciousness as created by Electromagnetic Force. International Journal of Applied and Advanced Scientific Research (IJAASR) 4(1): 1-6.

4. Di Sia P, Bhadra NK (2020) Origin of consciousness and contemporary physics. World Scientific News (WSN) 140: 127-138.

5. Di Sia $P(2018) D=4, N=1$ supergravity in superspace: general overview and technical analysis. World Scientific News 94(1): 1-71.

6. Hiramatsu T, Kawasaki M, Saikawa K (2011) Evolution of string-wall networks and axionic domain wall problem. Journal of Cosmology and Astroparticle Physics 8: 30.

7. Baez JC, Huerta J (2010) The Algebra of Grand Unified Theories. Cornell University, arXiv:0904.1556v2 [hepth].

8. Grozman P, Leites D, Shchepochkina I (2005) Lie superalgebras of string theories. Acta Mathematica Vietnamica 26: 27-63.

9. Beiser (2003) Concepts of Modern Physics, $6^{\text {th }}$ (Edn.), McGraw-Hill, Boston.

10. Di Sia P (2018) Mindfulness, Consciousness and Quantum Physics. World Scientific News (WSN) 96: 25-34.

11. Wilson CM, Johansson G, Pourkabirian A, Simoen M, Johansson JR, et al. (2011) Observation of the dynamical Casimir effect in a superconducting circuit. Nature 479(1): 376-379.

12. Rizzuto L, Spagnolo S (2009) Lamb shift of a uniformly accelerated hydrogen atom in the presence of a conducting plate. Physical Review A 79: 062110.

13. Harber DM, Obrecht JM, McGuirk JM, Cornell EA (2005) Measurement of the Casimir-Polder force through centerof-mass oscillations of a Bose-Einstein condensate. Physical Review A 72: 033610.

14. Vuillermet G, Gires PY, Casset F, Poulain C (2016) Chladni Patterns in a Liquid at Microscale. Physical Review Letters 116: 184501.

15. Jenny H (2007) Cymatics: A Study of Wave Phenomena \& Vibration. Macromedia, Cesena.
16. Di Biase F (2009) Quantum-holographic informational consciousness. Neuro Quantology 7(4): 657-664.

17. Susskind L (1995) The World as a Hologram. Journal of Mathematical Physics 36(11): 6377-6396.

18. Smolin L (2017) Three Roads to Quantum Gravity, Basic Books, New York.

19. Aharony O, Gubser S, Maldacena J, Ooguri H, Oz Y (2000) Large N Field Theories, String Theory and Gravity. Physics Reports 323(3-4): 183-386.

20. Maldacena J (2014) The gauge/gravity duality. Cornell University, arXiv:1106.6073v2 [hep-th].

21. Einstein, Podolsky B, Rosen N (1935) Can QuantumMechanical Description of Physical Reality Be Considered Complete? Physical Review 47: 777.

22. Di Sia P (2020) On philosophy of mind, quantum physics and metaphysics of the uni-multiverse. Philosophical News (in press).

23. Wheeler JA (1989) Information, physics, quantum: the search for links. Proceedings III International Symposium on Foundations of Quantum Mechanics, Tokyo, pp: 354-368.

24. Bohm D, Hiley BJ (1993) The Undivided Universe: An Ontological Interpretation of Quantum Theory, $1^{\text {st }}$ (Edn.), Routledge, London and New York.

25. Vedral V (2010) Decoding Reality. The Universe as Quantum Information. Oxford University Press, Oxford.

26. Pribram KH (1999) Quantum Holography: Is it Relevant to Brain Function? Information Sciences 115(1-4): 97102.

27. Di Sia P (2014) Relativistic nano-transport and artificial neural networks: details by a new analytical model. International Journal of Artificial Intelligence and Mechatronics (IJAIM) 3(3): 96-100.

28. Di Sia P (2018) Quantum-Relativistic Velocities in NanoTransport. Applied Surface Science 446(1): 187-190.

29. Di Sia P (2019) Mathematics and Physics for Nanotechnology-Technical Tools and Modelling. Jenny Stanford Publishing, CRC Press, Taylor \& Francis, Singapore. 